\title{
Performance analysis of the bump beams made of High Strength Steel and Carbon Fiber reinforce plastics
}

\author{
Yi Yao ${ }^{1}$, Quan Yuan ${ }^{2,3, a}$ *, Sihuan Fu² 4 \\ ${ }_{1}^{1}$ Pan Asia Technical Automotive Center Co., Ltd. Shanghai 201206, China \\ ${ }^{2}$ School of Automotive Engineering, Wuhan University of Technology, Wuhan 430070, China \\ ${ }^{3}$ Hubei Key Laboratory of Advanced Technology for Automotive Components, Wuhan 430070, China \\ ${ }^{4}$ SAIC GM Wuling Automobile Co., Ltd, Liuzhou 545007, China \\ a* Corresponding author, email:864177940@qq.com
}

\begin{abstract}
Lightweight and safe are the key research directions of the automobile industry. Bump beam is an important part of the car safety protection and it's lightweight design has drawn much attention. In this paper, the static strength and the low-speed impact performances of two types of bump beam with the same structure, which made of carbon fiber reinforced plastics (CFRP) and high strength steel (HSS), were investigated. Under the condition of static press, frontal and $40 \%$ offset impact, part structural stiffness, component crush intrusion, energy absorption were comparison analyzed. In order to guide the product design, the deformation and failure of CFRP beams with five different laminate structures were simulated under frontal and $40 \%$ offset impact. The results indicate that, with the same structure, the properties of CFRP bump beam are generally superior to HSS product, more conducive to lightweight and the optimum design of the laminate is $\left[0 \% / 60^{\circ} / 120^{\circ}\right]$
\end{abstract}

\section{Introduction}

Body lightweight and safety is an important aspect of automotive technology [1]. Bump beam is one of the main crash safety structures in the front of the car, and its lightweight technology has drawn wide attention ${ }^{[2]}$.

In recent years, a large number of researchers have carried out research work on the crashworthiness and lightweight design of vehicle bump beam. M.M. Davoodi Ramin Hosseinzadeh and Zhang Haiyang etc ${ }^{[3,4,5]}$ studied the structural design and composite design of lightweight bump beam. The results show that, for satisfying the requirements of the crashworthiness performance, lightweight effect is more obvious when use Composites; Dong-Kyou Park and Zhang Jing wen etc ${ }^{[6,7]}$ established a numerical analysis model to analyze the performance of HSS bump beam; Sun Huiwei etc ${ }^{[8]}$ conducted simulation analysis to the structure of the bump beam, put forward a structural improvement program to optimize the product performance; Hong Qiucai etc ${ }^{[9]}$ with the acceleration wave form, front bulkhead intrusion and the deformation pattern of front side rail as the development objective of structure, the optimization design of bump beam and body parts is carried out.

Due to carbon fiber composite materials in the obvious advantages of lightweight, it has broad application prospects in the automotive industry ${ }^{[10]}$. In order to explore the design and development of 
lightweight bumper, this paper comparatively studied the crashworthiness of bump beam which made of CFRP and HSS, and simulated the performance of CFRP bump beam with different plies design, for reference in engineering design.

\section{Set the constraints conditions}

The main analysis of this paper is the static load and the regular pattern of the low-speed crash of the same type of bump beam in different materials. Thus using a simplified model of the bump beam, the thickness is 3.34 $\mathrm{mm}$. Based on experience and calculation, the mesh size is initially determined to be $10 \mathrm{~mm}$, while some key sections is controlled to $5 \mathrm{~mm}$.

The high strength steel is selected No. 20 material model from the LS-DYNA material library ${ }^{[11]}$, the composite material selected carbon fiber material No. 54 model.

Collider intrusion can not exceed the allowable intrusion. In this paper, the size of the bump beam sets the maximum intrusion amount of the impact beam to be $50 \mathrm{~mm}$ when the low-speed frontal impact, and the low-speed offset impact value should be $25 \mathrm{~mm}$.

In the static analysis, the loading on the red part is given a total of 8 points with a load of $200 \mathrm{~N}$ at each point, while the yellow part shows the constraint. The point of the 6 degrees of freedom all constraints (as shown in Fig. 1 (a))and using the same constraints and loads for static analysis of two types of bump beams, which made of high strength steel and carbon fiber composite. In low-speed frontal and $40 \%$ offset impact, in order to simplify the process of solving the problem, the following only select the bump beam system for analysis, extraction of bump beam and energy-absorbing box as the crash analysis object, set the boundary conditions of the bump beam 6 degrees of freedom constraints. And giving the collider a mass of $1200 \mathrm{~kg}$ and constraint the degree of freedom of rotation of the collider about these three axes (as shown in Fig. 1(b) and(c)). Set the crash speed $4 \mathrm{~km} / \mathrm{h}$, when occurred frontal impact; Under the condition of $40 \%$ offset impact, the collider crashed into the bump beam at a speed of $2.5 \mathrm{~km} / \mathrm{h}$.

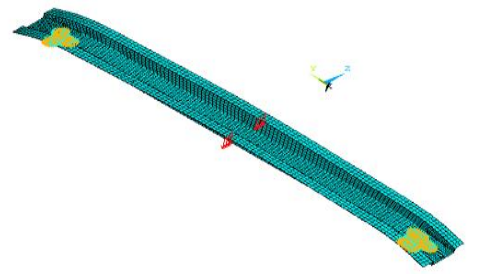

(a)

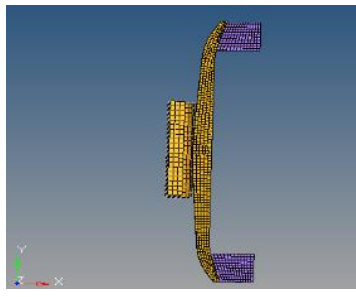

(b)

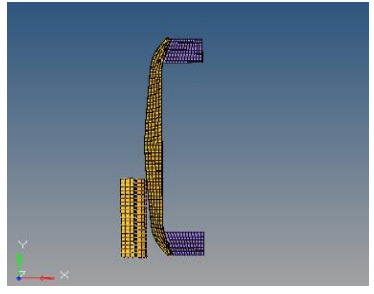

(c)
Figure 1 Constraints and Loads in three Conditions
(a): static load;
(b) frontal impact;
(c) $40 \%$ offset impact

\section{Results and analysis}

\subsection{Static Load Conditions}

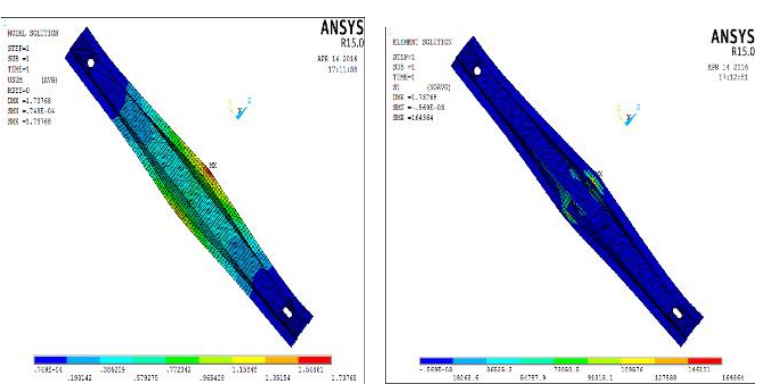

Figure 2 Maximum displacement cloud and stress distribution of CFRP bump beam

Due to the two materials are different, the stiffness is usually stress analysis, the results of larger errors. The general principle of equal stiffness to be analyzed, the maximum displacement as the research object for analysis. It can be seen from the Fig.2 that the maximum displacement of the HSS bump beam is $1.84 \mathrm{~mm}$ and the maximum displacement of the CFRP bump beam is 1.74 $\mathrm{mm}$. Under the same constraint and load conditions, the CFRP bump beam not only reduces the weight by $64.12 \%$, but also reduces the displacement.

\subsection{Frontal Impact}

Displacement comparision chart of high strength 
steel bump beam and carbon fiber composite beam under frontal impact are shown in Fig.3. The maximum deformation of two kinds of bump beam are $44.35 \mathrm{~mm}$ and $41.12 \mathrm{~mm}$ respectively, which are less than the allowable displacement of $50 \mathrm{~mm}$ and meet the requirements. However, the displacement of carbon fiber composite material smaller, more superior performance.

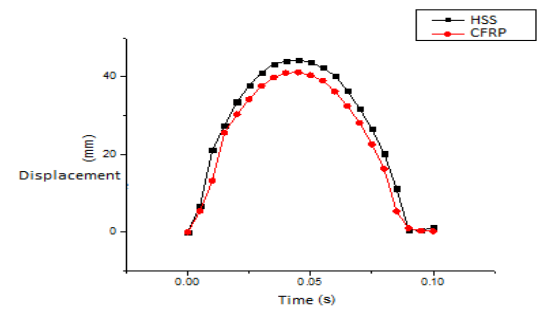

Figure 3 Frontal Impact Displacement Comparison Chart

Fig.4 shows the variation of system energy under frontal impact conditions. The total energy of the system is equal to the initial kinetic energy of the collider being $550 \mathrm{~J}$ and remains roughly the initial value during the collision. During the collision, the kinetic energy was continuously transformed into the internal energy of the system, about $42 \mathrm{~ms}$, the internal energy of the two materials reached the peak values, respectively $548 \mathrm{~J}$ and $547 \mathrm{~J}$. The proportion of total energy is roughly the same and with a small difference. Subsequently, the internal energy continued to decrease. At $90 \mathrm{~ms}$, the elastic deformation of bump beam has basically recovered. The internal energy and kinetic energy were maintained at a stable value.

Although the energy curves of the two materials are almost the same, the numerical difference is small, but the quality of the carbon fiber composite material bump beam is far less than the high strength steel bump beam, resulting in large difference between specific energy absorption, as shown in Fig.5. The specific energy absorption of composite material is $109 \mathrm{~J} / \mathrm{kg}$, while the high strength steel's specific energy absorption is only $50 \mathrm{~J} / \mathrm{kg}$.

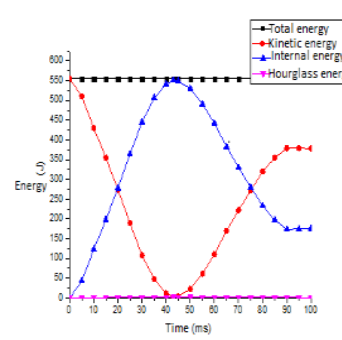

(a)

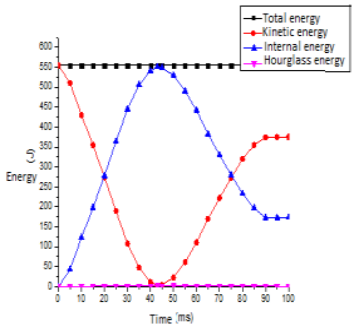

(b)
Figure 4 Frontal Impact Energy Change Diagram (a)HSS; （b ) CFRP

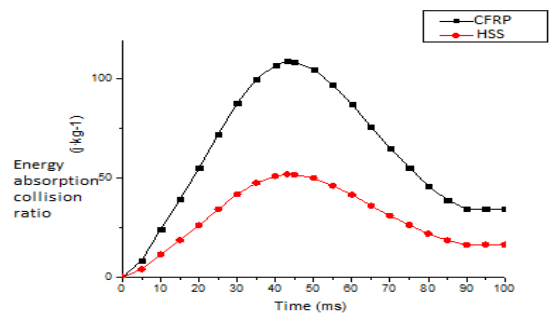

Figure 5 Frontal Impact of Two Materials Specific Energy Absorption Comparison Chart

\section{$3.340 \%$ Offset Impact}

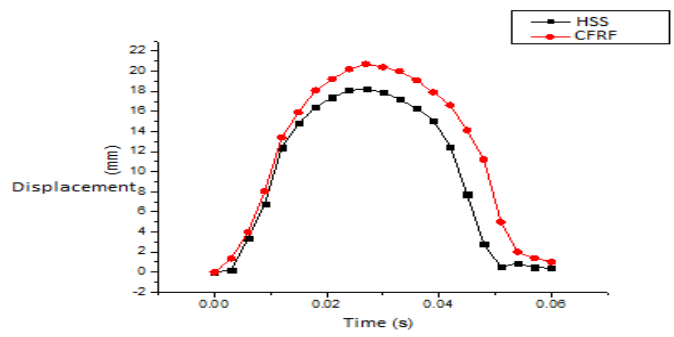

Figure 6 40\% Offset Impact Displacement Comparison Chart

As shown in Fig.6, the maximum deformation of high strength steel bump beam and carbon fiber composite beam are $18.35 \mathrm{~mm}$ and $20.92 \mathrm{~mm}$, respectively, which are less than the allowable displacement of $25 \mathrm{~mm}$, meet the requirements. But the deformation of carbon fiber composite material is slightly larger.

In $40 \%$ offset impact condition, the energy curves of various parts of the system over time are roughly the same as the frontal impact. The total energy of the system is equal to the initial kinetic energy of the collider is about $221 \mathrm{~J}$, and the value is basically maintained during the collision. During the collision, the kinetic energy was continuously transformed into the internal energy of the system, about $23 \mathrm{~ms}$, the internal energy of the two materials reached the peak values, respectively $219 \mathrm{~J}$ and 
$218 \mathrm{~J}$. The proportion of total energy is roughly the same, simultaneously, the energy of structure difference is minimal; Then, the bump beam system continuously changes back to the initial shape, and the internal energy decreases. At $43 \mathrm{~ms}$ of collision, the deformation of the bump beam disappears, and the internal energy and kinetic energy remain at a certain value. However, the specific energy absorption of carbon fiber composites is $42 \mathrm{~J} / \mathrm{kg}$, higher than the specific energy absorption of high strength steel $21 \mathrm{~J} / \mathrm{kg}$.

\section{Stacking Design of CFRP Bump Beam}

Before analyzing the bump beam layer structure, the multi-structural analysis of static load condition determines a fixed laminate thickness to simplify the analysis process and avoid the complexity of the analysis process and increase the analysis time due to the change of the number of layers. In general, ply orientation of $0^{\circ}$ carry axial load, $\pm 45^{\circ}$ carry shear loads, and $90{ }^{\circ}$ carry lateral loads and control Poisson's ratio[12]. Therefore, according to the comparative analysis of the above two materials, under the condition that the number of layers of carbon fiber composite material is 20 , applying constraint and load according to the different working conditions of the beam in accordance with the comparative analysis of the beam with high strength steel and carbon fiber composite material by changing stack sequence.

In this paper, selecting stack sequence are layer1 $\left[0^{\circ} / 90^{\circ}\right] \mathrm{nt}, \quad$ layer $2\left[0 \% \% 0^{\circ} / 120^{\circ}\right] \mathrm{nt}, \quad$ layer 3 $\left[0^{\circ} / 90^{\circ} / 45^{\circ} /-45^{\circ}\right] \mathrm{nt}, \quad$ layer4 $\left[45^{\circ} /-45^{\circ}\right] \mathrm{nt}$, layer 5 $\left[0 \% / 45^{\circ} /-45^{\circ}\right]$ nt.

In the frontal and $40 \%$ offset impact conditions, the maximum displacement and stress curve under five kinds of layer design, is shown in Fig.7 and Fig.8:

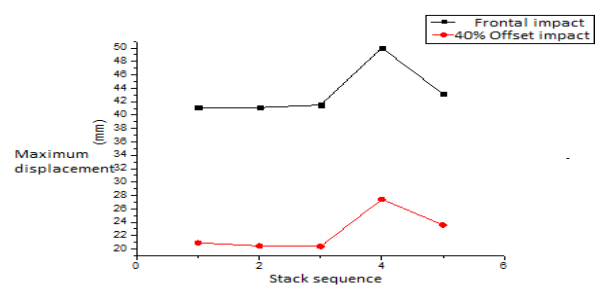

Figure 7 Frontal and 40\%Offset Impact Maximum

Displacement Comparison Chart

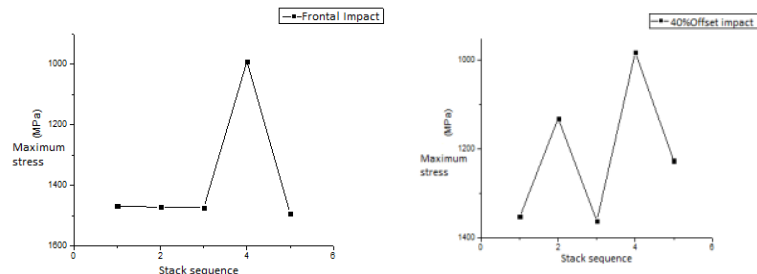

Figure 8 Frontal and 40\%Offset Impact Maximum Stress Comparison Chart

From the comparison of the above graphs, we can see that for the maximum displacement, under the conditions of low-speed frontal and $40 \%$ offset impact, the maximum displacement changes little with stack sequence, except for the layer order 4 and the maximum displacement is smaller at $\left[0^{\circ} / 90^{\circ}\right],\left[0^{\circ} / 60^{\circ} / 120^{\circ}\right]$ and $\left[0^{\circ} / 45^{\circ} /-45^{\circ} / 90^{\circ}\right]$. For the maximum stress, under the low-speed frontal impact, the maximum stress of layer 4 hardly changes with stack sequence, and the maximum stress reaches the minimum under the layer of $\left[45^{\circ} /-45^{\circ}\right]$. Under the condition of $40 \%$ offset impact, from the analysis results, it can be seen that the maximum stress of other lay-up sequences is basically the same except that the maximum stress of the layer of $\left[0^{\circ} / 60^{\circ} /\right.$ $\left.120^{\circ}\right]$ and $\left[45^{\circ} /{ }^{\circ}-45^{\circ}\right]$ are smaller. Therefore, the optimal lay-up order is $\left[0^{\circ} / 60^{\circ} / 120^{\circ}\right]$.

\section{Summary}

(1) In the same structure, CFRP bump beam is superior to HSS bump beam in terms of static stiffness, crash intrusion, energy absorption and specific energy absorption to meet the performance requirements of the beam, and the quality is lighter.

(2) Among the various layer design schemes that meet the static stiffness and low-speed collision conditions, the optimal lay-up order is $\left[0^{\circ} / 60^{\circ} / 120^{\circ}\right]$.

\section{References}

1. Society of Automotive Engineers of China. Trace research on world automotive technology development (lightweight) [M]. Beijing: Beijing Institute of Technology Press, (2013).

2. Z.G. An, Y.Y. Zhao, The structural strength design of the light weight of the car crash beam of 
anti-collision beam [J]. Computer Simulation, 33, 7 (2016)

3. M.M. Davoodi, S.M. Sapuan, D. Ahmad. Concept election of car bumper beam with developed hybrid bio-composite material [J]. MATER DESIGN, 32, 9 (2011)

4. Ramin, Hosseinzadeh, Mahmood M. Ramin Hosseinzadeh, Larry B.Lessard. Parametric study of automotive composite bumper beams subjected to low-velocity impacts [J]. COMPOS STRUCT, 68, 9 (2005)

5. H.Y. Zhang, H. Fu, Z. Xiao, B. Li, S.Y. Duan. Lightweight research on bumper beam of fiber reinforced composite [J]. FIBER COMPOS, 10, 7 (2017)

6. D.K. Park. A development of simple analysis model on bumper barrier impact and new IIHS bumper impact using the dynamically equivalent beam approach[J]. J MECH SCI TECHNOL, 25, 8 (2011)

7. J.W. Zhang, T.Q Fan, Q.J. Zhao, Y. Li. Crash performances comparison and selection of bumper beam with different lightweight methods [C]. Proceedings of the 11th International Forum of Automotive Traffic Safety, (2014)

8. H.W. Sun, X.C. Zhang, Z.F, Liu, Y. Xin, Z. Yang Optimization of front anti-collision beam structure in offset crash [J]. AUTO ENG, 6,4,(2017)
9. Q.C. Hong, W.G, Liu, D.Y. Zhou, A Study on Body Structure Lightweighting in the Transfiguration Development of a Sedan [J]. AUTOMOT ENG, 39, 5,(2017)

10. X.P. Yang, Z.B. Huang, Z.Y, Zhang, H.P. Yang, Y.J. Zhou, Application progress of carbon fiber reinforced polymers for energy saving and emission reduction [J]. MATER REV, 5 (2010)

11. Z.Y. Hu, B.Q. Zeng, S.G. Xie. Vehicle Safety Simulation and Analysis Based on LS-DYNA and HyperWorks [M]. Beijing: Tsinghua University Press (2011)

12. J. Zhang. Structural Analyses and Layer Optimization of Large Scale Wind Turbine Blade [D].Wuxi: Jiangnan University, (2013) 